\title{
HABILIDADES SOCIAIS EDUCATIVAS PARA MÃES DE CRIANÇAS COM TDAH E A INCLUSÃO ESCOLAR
}

\author{
Educational social skills for mothers of children with ADHD \\ and the school inclusion
}

\begin{abstract}
Margarette Matesco Rocha ${ }^{[a]}$ Zilda Aparecida Pereira Del Prette ${ }^{[b]}$
${ }^{[a]}$ Doutora em Educação Especial pela Universidade Federal de São Carlos (UFSCar), professora do Departamento de Psicologia Geral e Análise do Comportamento da Universidade Estadual de Londrina (UEL), Londrina, PR - Brasil, e-mail: margarette@uel.br

[b] Pós-doutorado na Universidade da Califórnia, professora titular da Universidade Federal de São Carlos (UFSCar), vinculada ao Programa de Pós-Graduação em Educação Especial e ao de Psicologia, São Carlos, SP - Brasil, e-mail: zdprette@power.ufscar.br
\end{abstract}

\section{Resumo}

Este artigo apresenta o Transtorno do Déficit de Atenção e Hiperatividade (TDAH) como uma categoria da Educação Especial, focalizando as implicações desse transtorno no ambiente escolar e o papel dos pais na parceria família-escola. Destaca as habilidades pessoais e parentais que os pais de crianças com TDAH devem apresentar com vistas a ações educativas mais efetivas, contribuindo para a promoção do desenvolvimento das habilidades sociais e acadêmicas de seus filhos e possibilitando que eles desfrutem as oportunidades sociais e educacionais promovidas pelo ambiente escolar. Essas habilidades representam um conjunto de ações potencialmente eficazes para estabelecer condições, no ambiente familiar, para a modificação do comportamento da criança. Defende a participação da família para a inclusão escolar, mas não lhe imputa a responsabilidade por esse processo, nem reduz o papel dos professores nessa tarefa. Situa a família como componente ativo e essencial à educação (inclusive escolar) dos filhos e a importância da parceria com a escola para enfrentar os desafios impostos à aprendizagem e ao desenvolvimento da criança com TDAH. Além disso, considerando a grande variabilidade na forma e no comportamento individual dessas crianças em vários contextos, defende a promoção de uma abordagem educacional baseada nas necessidades educacionais específicas de cada estudante com TDAH e não na categoria genérica de Educação Especial na qual o indivíduo foi classificado.

Palavras-chave: Habilidades sociais. TDAH. Educação especial. 


\begin{abstract}
This essay presents the Attention Deficit Hyperactivity Disorder (ADHD) as a category of the Special Education, focusing on the implications of this disorder in school's environment and on the role of the parents in the family-school partnership. It highlights the personal and parental skills which the parents of children with ADHD have to present regarding the most effective educational actions, contributing for the promotion of the development of their kids' social and academic skills and enabling them to enjoy the social and educational opportunities promoted by the school environment. These skills represent a group of potentially effective actions for establishing the conditions, in the family environment, for the child's behavior modification. It defends the family participation for the school inclusion, but doesn't impose them the responsibility for this process or reduce the teacher's role on this task. It situates the family as an active component and essential to the kids' education (school education included) and the importance of the partnership with the school to face the challenges imposed to the learning and the development of the child with ADHD. Furthermore, considering the great variability in the form and in the individual behavior of these children in several contexts, it defends an educational approach based on actual educative need of each student with ADHD and not on the generic category of Special Education in which the individual has been qualified.
\end{abstract}

Keywords: Social skills. ADHD. Special education.

\section{INTRODUÇÃO}

Atualmente existem inúmeras publicações nacionais e internacionais que retratam as diversas características do Transtorno de Déficit de Atenção e Hiperatividade/Impulsividade (TDAH). Esses estudos, apoiados em evidências empíricas, trazem informações relevantes sobre sua caracterização e análises atualizadas de descobertas relativas ao transtorno, com o objetivo de favorecer um entendimento de suas várias dimensões. No entanto, são raros os trabalhos brasileiros que discutem o TDAH como uma categoria da Educação Especial e, principalmente, a preparação das famílias de crianças com TDAH para se tornarem agentes efetivos no processo de inclusão escolar de seus filhos.

Assim, esse trabalho busca apresentar o TDAH como uma categoria da Educação Especial e retratar as habilidades pessoais e parentais que pais de crianças com TDAH devem apresentar com vistas a ações educativas mais efetivas, contribuindo para a promoção do desenvolvimento das habilidades sociais e acadêmicas de seus filhos e possibilitando que eles desfrutem as oportunidades sociais e educacionais promovidas pelo ambiente escolar.

\section{TDAH e Educação Especial}

O Transtorno de Déficit de Atenção e Hiperatividade/Impulsividade (TDAH) caracterizase, essencialmente, por um padrão persistente de desatenção, hiperatividade e impulsividade, mais frequente e severo do que habitualmente observado em indivíduos com um nível de desenvolvimento comparável (Manual Diagnóstico e Estatístico de Transtornos Mentais [DSM- IV-TR], 2002). A manifestação concomitante dos comportamentos de desatenção e hiperatividade/impulsividade não é comum a todas as crianças diagnosticadas com TDAH. Algumas demonstram somente comportamentos relacionados ou à desatenção ou à hiperatividade/impulsividade. Essas diferentes manifestações são classificadas pelo DSM-IV como subtipos do TDAH, com base no predomínio de sintomas de desatenção (tipo predominantemente desatento) ou sintomas de hiperatividade e impulsividade (tipo predominantemente hiperativoimpulsivo) ou de ambos (tipo combinado).

Além das várias formas de apresentação e subtipos do TDAH, estima-se que mais de 50\% das crianças com TDAH apresentam comorbidades com outras patologias (Condemarín, Gorostegui \& Milicic, 2006; Rodhe \& Benczik, 1999). Isso 
significa que, além do comprometimento associado aos sintomas básicos de desatenção, hiperatividade e impulsividade, essas crianças e adolescentes com significativa frequência podem apresentar comprometimentos adicionais decorrentes da comorbidade com outros transtornos psiquiátricos. Os principais tipos de comorbidade (Souza \& Pinheiro, 2003) são: Transtorno Desafiador de Oposição (TDO), Transtorno de Conduta (TC), Transtornos de Tiques, Transtornos de Ansiedade, Transtornos do Humor (depressivo e bipolar) e Transtornos do Aprendizado e da Linguagem. Esses transtornos aumentam potencialmente o comprometimento funcional de crianças e adolescentes com TDAH (Souza, Serra, Mattos, \& Franco, 2001) e afetam de modo significativo a expressão dos sintomas, o prognóstico e a resposta ao tratamento (Condemarín et al., 2006).

Com relação ao diagnóstico, Condemarín et al. (2006) destacam que as diferentes características e sintomas apresentados pelas crianças com TDAH torna difícil o diagnóstico e remete à necessidade de avaliações mutimodais e multidisciplinares. Para DuPaul e Stoner (2007), os múltiplos métodos de avaliação conduzidos em vários ambientes devem ser utilizados para determinar os comportamentos problemáticos específicos da criança, as variáveis controladoras e as possíveis estratégias de intervenção aplicáveis para cada criança. De forma geral, os principais instrumentos de avaliação incluem entrevistas e questionários, a serem respondidos pelos pais e professores, e observações do comportamento da criança em múltiplos contextos e sob variadas condições para a tarefa (DuPaul \& Stoner, 2007).

Embora seja um dos transtornos mais comuns na infância (Barkley, 2002; Sena \& Neto, 2007), ainda não há um acordo acerca da prevalência exata do TDAH na população mundial. Essa falta de consenso é decorrente das diferenças metodológicas entre os estudos com relação à representatividade e tamanho da amostra (Petersen, Bilenberg, Hoerder \& Gillberg, 2006), aos diferentes tipos de amostra (clínicas ou populacionais) e idade das crianças participantes dos vários estudos (Golfeto \& Barbosa, 2003), à utilização de diferentes tipos de entrevistas - estruturada, semiestruturada ou livre-, aos delineamentos de estudos (Golfeto \&
Barbosa, 2003; Petersen et al., 2006), às várias definições adotadas, devido ao uso de sistemas diagnósticos diferenciados (principalmente o DSM e o CID) e ao critério para definição, que pode ou não incluir os prejuízos funcionais (Petersen et al., 2006), e ao uso de diferentes fontes de informação, como pais, professores ou o próprio indivíduo (Golfeto \& Barbosa, 2003).

Da mesma forma, também não há dados sobre a prevalência do TDAH no país, porém, a partir de diversos estudos (Freire \& Pondé; 2005; Guardiola, Fuchs, \& Rotta, 2000; Poeta \& Neto, 2004; Rohde et al., 1999, Vasconcelos et al., 2003), em diferentes cidades brasileiras, pode-se estimar que no mínimo 3,5\% das crianças brasileiras em idade escolar apresentam o transtorno, que ocasiona efeitos devastadores na sua vida familiar, social e nos estudos, sendo, portanto, um tema merecedor de discussões em diversas áreas de conhecimento, dentre elas a Educação Especial.

Os dados a respeito da proporção de crianças com TDAH nos programas de Educação Especial também não foram estimados, e os disponíveis referem-se ao número de alunos matriculados na Educação Especial sob a rubrica de condutas típicas. No ano de 2006 , crianças com condutas típicas ocupavam o segundo lugar em termos de matrículas na Educação Especial e frequentavam, em sua grande maioria, escolas regulares/classes comuns. O número de matrículas no ensino superior demonstra que cerca de 450 alunos com condutas típicas frequentavam, em 2006, essa etapa/modalidade de ensino (Brasil, 2006), que corresponde aproximadamente a $0,01 \%{ }^{1}$ do total de matrículas no ensino superior presencial no Brasil.

Os atuais critérios de definição da clientela da Educação Especial estão descritos nas Diretrizes Nacionais para a Educação Especial na Educação Básica (Resolução 2/2001). De acordo com esse documento, tal clientela é constituída por três grandes grupos, cada qual reunindo tipos e graus de necessidades educacionais especiais (Brasil, 2001). Um dos grupos refere-se àqueles estudantes com dificuldades acentuadas de aprendizagem ou limitações no processo de desenvolvimento, podendo estar vinculado a condições, disfunções, limitações ou deficiências ou sem vinculação a uma

\footnotetext{
Proporção obtida a partir do número total de matrículas (4.676.646) no ensino superior brasileiro (presencial), constatada pelo Censo da Educação Superior realizado pelo INEP (Instituto Nacional de Estudos e Pesquisas Educacionais Anísio Teixeira) em 2006.
} 
causa orgânica específica, o que dificulta o acompanhamento das atividades curriculares.

É dentro desse grupo que se encontram os alunos com dificuldades de adaptação escolar por manifestações de condutas típicas, definidas como "dificuldades causadas por comportamentos que tendem a prejudicar, e por vezes inviabilizar, as relações do aluno com seu professore/ou com seus colegas, com os materiais de uso pessoal e coletivo e ainda o processo ensino-aprendizagem" (Brasil, 2002a, p. 15). Na perspectiva dos documentos oficiais (Brasil, 2002a; 2002b), alunos com sintomas primários (desatenção e hiperatividade) do TDAH são denominados "alunos com condutas típicas" por apresentarem dificuldades acentuadas de aprendizagem associadas às condutas típicas de síndromes de quadros neurológicos. Nesses documentos, a referência aos alunos com TDAH é feita com relação aos comportamentos de desatenção e hiperatividade apresentados poresses alunos, não sendo, portanto, utilizados a expressão Transtorno do Déficit de Atenção e Hiperatividade ou seu acrônimo TDAH.

De forma mais explícita, são crianças que não conseguem focar a atenção, isso é, frente a um conjunto de estímulos aos quais são expostos, não conseguem selecionar o que é relevante e o que deve serignorado e, em consequência, seu processo de aprendizagem, acadêmico ou social, é prejudicado (Hübner \& Marinotti, 2000). Considerando o modelo comportamental de autocontrole, supõe-se que o comportamento de indivíduos com TDAH é afetado principalmente por consequências imediatas, e que as atrasadas têm sua função reduzida, caracterizando-se, dessa forma, o conjunto de respostas que descreve os comportamentos de impulsividade/hiperatividade observados nesses indivíduos (Hoerger \& Mace, 2006; Neef, Marckel \& Ferreri, 2005).

Assim, cabe ressaltar que a Educação Especial se refere a todas as crianças que enfrentam barreiras de acesso à escolarização ou de acesso ao currículo, que levam ao fracasso escolar e à exclusão social (Ferreira, 2005), como é o caso das crianças com TDAH. Além disso, um dos pressupostos básicos da Educação Especial é o questionamento da "patologização" do comportamento e a defesa da necessidade das escolas flexibilizarem suas expectativas e se prepararem pedagogicamente para receber alunos com necessidades educacionais especiais na sua prática diária, diferentemente de especialistas no transtorno biológico, os quais acentuam os fatores genéticos e prescrevem tratamentos voltados para a redução dos sintomas e adaptação do indivíduo a padrões de comportamento preestabelecidos (Landskron \& Sperb, 2007).

Com relação à educação formal, as crianças com TDAH constituem, reconhecidamente, um grupo de crianças que apresenta necessidades educacionais diferenciadas e que, portanto, requer um atendimento educacional especializado. As características peculiares por elas apresentadas, tais como o breve período de atenção, o alto nível de atividade e o limitado controle de impulsos, restringem sua capacidade de atender às demandas exigidas pela escola nas atividades acadêmicas e sociais. Por exemplo, o alto nível de atividade pode desviar a atenção da criança às instruções e, assim, minimizar a "conquista" da informação acadêmica, ou elas podem tomar decisões apressadas demais nas tarefas, prejudicando seu desempenho independente (DuPaul \& Stoner, 2007).

Diferentemente de alguns grupos de crianças que estão em escolas especiais, as crianças com TDAH não estão fora do ensino regular, mas podem constituir um grupo de risco para a Educação Especial. Na concepção de Ferreira (2005; 2006), essas crianças têm acesso à educação - melhor seria dizer à escola -, mas com muita frequência encontram-se em permanente risco de exclusão, por razões distintas, entre as quais a crença de que não são capazes de aprender e o fato de não corresponderem às expectativas dos professores e pais nas atividades escolares. Por fatores como esses, são expulsos ou suspensos e experimentam continuamente a discriminação e/ ou maus tratos, tanto na família como na escola.

A perspectiva de uma educação inclusiva, que preconiza a valorização da diversidade bem como a igualdade de direitos e oportunidades educacionais para todos, enfatiza as possibilidades de aprendizagem e não as limitações das crianças com necessidades educacionais especiais. Essa postura implica, portanto, em reconhecer as falhas do ambiente social, proporcionando condições adequadas às suas necessidades de aprendizagem e desenvolvimento, e não destacando suas limitações ou deficiências (Glat \& Fernandes, 2005).

No caso específico do TDAH, essa consideração torna-se fundamental, uma vez que os critérios de avaliação foram desenvolvidos dentro de um contexto médico, sugerindo que o problema está localizado na criança enquanto "portadora" de um transtorno e negligenciando as 
variáveis ambientais que poderiam exercer um papel na manutenção ou agravamento dos comportamentos apresentados pela criança (DuPaul \& Stoner, 2007). De modo similar, Cypel (2007) afirma que se referir somente à criança e ao seu distúrbio de comportamento, sem alusão ao seu meio ou ao contexto de vida, é desconsiderar as influências que o ambiente social exerce sobre o seu desenvolvimento, atribuindo-se à criança toda a responsabilidade pelo quadro apresentado.

Ainda nesse sentido, o uso dos termos "TDAH" ou "crianças com TDAH" não deve ser utilizado como explicação do comportamento nem para justificar os desempenhos apresentados por essas crianças, até porque o rótulo não traz em si qualquer explicação para o fenômeno comportamental, que é sempre muito complexo. Ao invés disso, deviam ser usados para identificar formas predominantes de desempenhos (sociais e escolares) de um grupo de crianças que apresenta um estilo próprio de aprendizado, em situação formal e informal, e com o propósito primordial de orientar e de discutir as ações educativas necessárias a esse grupo de crianças. Isso não implica desconsiderar a necessidade de intervenção medicamentosa, que comprovadamente (Multimodal Treatment Study of Children with ADHD [MTA], 1999) auxilia na atenuação das manifestações próprias do quadro de TDAH, mas sim em validar a necessidade de intervenções psicoeducativas combinadas que envolvem a própria criança, a escola e a família.

\section{TDAH, inclusão e papel da família}

Para Condemarín et al. (2006), as intervenções no ambiente familiar e escolar justificam-se pelo fato do quadro da criança com TDAH poder evoluir para formas mais positivas ou então piorar, a depender das condições que lhe são oferecidas. Assim, a qualidade dos contextos escolar e familiar pode constituir fator determinante para a forma como a criança vive suas dificuldades e os mecanismos que utiliza para superá-las. No caso específico das famílias, a análise da interação entre pais e filhos revela que esses pais demonstram estilo parental mais coercitivo ou aversivo (FinziDottan, Manor \& Tyano, 2006). Em tarefas de resolução de problemas são menos cativantes, calorosos, comprometidos e com estilo de comunicação menos efetivo (Tripp, Schaughency,
Langlands, \& Mouat, 2006) e apresentam, também, menos gratificações e diretrizes às crianças e mais coerção (Jonhson \& Mash, 2001). Além disso, exibem mais comportamentos diretivos, mais responsividade negativa e são menos positivos ao manejar os comportamentos dos filhos (Wells et al., 2000; Cunningham, 2007).

O estilo disciplinar dessas mães é considerado negligente por cederem mais facilmente aos apelos dos filhos, permitirem o descumprimento das regras e reforçar comportamentos inadequados (Jonhson \& Mash, 2001). Porém, quando as crianças não seguem as regras, as mães reagem com um parão caracterizado como raiva, irritabilidade e explosão (Banks, Ninowski, Mash, \& Semple, 2008). Todas essas características contribuem para que a interação entre pais e filhos com TDAH seja frequentemente mais conflituosa, e a vida da família, caracterizada pela desarmonia e discórdia (Wells et al., 2000).

Quando as práticas parentais são avaliadas pelos filhos (com TDAH), os resultados seguem praticamente a mesma direção. Rielly, Craig e Paker (2006) compararam as práticas parentais relatadas por 109 estudantes com TDAH e 109 sem TDAH, e os resultados evidenciaram que pais de crianças com TDAH apresentam mais práticas parentais negativas (por exemplo, disciplina inconsistente) do que positivas e que essas crianças experimentam mais feedbacks negativos de seus pais, além de pouca oportunidade de interações positivas, dentro e fora do lar, devido à falta de supervisão e monitoria dos pais.

A explicação comumente aceita, para a manutenção desses padrões de interações pais e filhos, sugere que eles ocorrem devido à história de insucesso dos pais para controlar os comportamentos da criança, o que pode tanto diminuir a frequência do uso de práticas parentais positivas (disciplina consistente, monitoria positiva) como favorecer o aumento de uso de práticas coercitivas (práticas disciplinares severas). A adoção dessas práticas educativas pode ocasionar ganhos positivos em curto prazo para os pais, porém terá custos muito maiores, em médio e longo prazo, para os pais e para crianças (Johnson \& Mash, 2001).

Deve-se considerar que as práticas parentais negativas podem ter início como uma reação à desatenção, impulsividade e hiperatividade apresentadas pela criança, como também pode ser decorrente de práticas educativas negativas já estabelecidas na 
interação familiar, portanto, sem uma relação inicial com as dificuldades apresentadas pela criança. No entanto, a manutenção do padrão parental caracterizado por punição excessiva e indiscriminada, ou de recompensa aos comportamentos inadequados ao invés dos adequados, influencia a maneira como a criança se comporta em relação aos seus pais, aumentando a frequência de comportamentos de rebeldia e oposição (Sena \& Neto, 2007), que, por sua vez, levam à manutenção das práticas educativas negativas.

Com relação aos recursos e déficits sociais apresentados por pais de crianças com TDAH, poucas investigações foram conduzidas. Adesida e Foreman (1999) e Neophytou e Webber (2005) verificaram que mães de crianças com TDAH isolam-se e isolam os filhos de contatos sociais devido ao alto nível de atenção e supervisão que essas crianças necessitam e também por apresentarem comportamentos que são fontes de embaraço e vergonha para os pais. Essas restrições envolvem frequentar lugares públicos como shoppings, restaurantes, cinemas, transportes públicos e fazer visitas a amigos e parentes. Frente essa situação, os pais passam, deliberadamente, a não frequentar muitas situações do dia-a-dia, julgandose inadequados em suas atitudes parentais e aumentando o seu isolamento social (McLaughlin \& Harrison, 2006; Adesida \& Foreman, 1999). Esse isolamento social tem como consequência práticas parentais menos efetivas (McLaughlin \& Harrison, 2006), aumentando a probabilidade de manutenção dos comportamentos inadequados dos filhos devido à falta de oportunidades e de modelos para a aprendizagem de comportamentos sociais mais efetivos (Del Prette \& Del Prette, 2005).

Assim, dentre as ações educativas necessárias, a defesa da necessidade de intervenção educativa na família dessas crianças é baseada na suposição de que o contexto social familiar pode proporcionar contingências favoráveis à aprendizagem de comportamentos incompatíveis com o padrão do TDAH e compatíveis com os comportamentos de estudar e de manter interações satisfatórias com pares e professores na escola. Essa escolha também se assenta na concepção de que um sistema inclusivo depende da participação e da parceria da família, a fim de garantir o acesso, a permanência e o sucesso dessas crianças na escola.

\section{TDAH e habilidades sociais educativas dos pais}

No caso das crianças com TDAH, a sua permanência no ambiente escolar depende, portanto, dessa rede de apoio para ampliar suas oportunidades de aprendizagem e garantir o desenvolvimento de suas capacidades acadêmicas ou sociais, que se encontram comprometidas. Nesse sentido, a família, entendida como um componente ativo, contribuinte e essencial à educação dos filhos, divide responsabilidades com a escola para enfrentar os desafios da escolarização impostos a essas crianças. Para essa tarefa, pais precisam apresentar um conjunto de ações que garanta a sua participação efetiva, enquanto educadores, em todos os contextos em que a criança está inserida, o que implica competências para interagir com filhos na promoção dos comportamentos exigidos nesses contextos.

Dentre as ações necessárias, Rocha (2009) identificou que pais de crianças com TDAH precisam de assessoria para o desenvolvimento de habilidades pessoais consideradas essenciais para a interação social, tanto no âmbito familiar como extrafamiliar, e de habilidades sociais específicas para proverem o desenvolvimento dos filhos. No caso das habilidades pessoais (de comunicação, de civilidade e assertivas), conforme descritas por Del Prette e Del Prette (2001), elas são necessárias para favorecer o aprendizado de interações positivas das mães com o filho e com as pessoas com quem interage cotidianamente por conta do problema da criança, bem como com as demais, sobre questões alheias aos comportamentos dos filhos, mas que podem ter implicações na interação com as crianças. Esse conjunto de comportamentos é essencial para elas lidarem com as demandas de interação com diferentes profissionais da saúde (médicos psiquiatras, neurologistas, fonoaudiólogos etc.), da educação (psicopedagogos, professores da sala de recursos, pessoal técnicoadministrativo das escolas etc.) e, ainda, com parentes próximos e vizinhança. Esses, em muitos casos, por nada ou pouco conhecerem sobre o TDAH, fornecem consequências punitivas aos comportamentos das mães e das crianças, gerando efeitos colaterais bastante danosos para a interação familiar. 
Por outro lado, déficits no repertório das mães para atuar frente às demandas interpessoais cotidianas, acrescidas das cobranças de várias fontes (marido, escola, parentes, outros filhos), no sentido de controlar os comportamentos dos filhos, representam um risco para a interação positiva com os filhos, favorecendo a adoção de práticas educativas coercitivas, sentimentos de incompetência parental e indícios de estresse, como identificados nos estudos da área (Chronis, Chacko, Fabiano, Wymbs, \& Pelham, 2004; Cunningham, 2007; Johnston \& Mash, 2001).

Com relação às habilidades parentais e a adaptação escolar das crianças com TDAH, esses pais precisam ser instrumentalizados em estratégias para aumentar a frequência de comportamentos acadêmicos importantes dos filhos, como copiar a tarefa de casa determinada pelo professor em sala de aula e, posteriormente, completá-la da melhor forma possível em casa. Além disso, mães precisam ser orientadas sobre estratégias que os filhos podem utilizar em reação a situações (bastante comuns) de provocações por colegas, o que é quase inevitável na vida social dessas crianças, provavelmente por reagirem mais impulsivamente às provocações, reforçando o comportamento do provocador.

Ainda dentre as habilidades, parentais Del Prette e Del Prette (2001) defendem que, como educadores, os pais devem apresentar um conjunto de habilidades sociais educativas visando a promover o desenvolvimento e a aprendizagem dos filhos, mesmo no contexto informal familiar. Essas habilidades representam um conjunto de ações potencialmente eficazes para estabelecer condições no ambiente familiar para a modificação do comportamento da criança. Quando os pais são bem-sucedidos, essas mudanças acabam funcionando também para manter esses comportamentos parentais efetivos.

Desse conjunto de ações (ver descrição detalhada em Del Prette \& Del Prette, 2009), considera-se que os pais de crianças com TDAH devem ser ensinados e incentivados, mais especificamente, a apresentar as habilidades de: prover feedback positivo, elogiar, incentivar, manifestar atenção ao relato da criança, obter informações, expressar discordância/reprovação e expressar concordância quando pertinentes, promover autoavaliação, arranjar ambiente físico de modo a explorar a ampliar oportunidades educativas, organizar materiais instrucionais, mediar interações da criança com outras, descrever/ justificar comportamentos desejáveis e indesejáveis, negociar regras, chamar atenção para normas preestabelecidas, pedir mudança de comportamento, apresentar instruções e dicas, parafrasear, resumir comportamentos emitidos e apresentar modelo. Essas e outras ações estão organizadas por Del Prette e Del Prette (2008) em quatro conjuntos mais gerais:

1) Estabelecer contextos interativos potencialmente educativos;

2) Transmitir ou expor conteúdos;

3) Estabelecer limites e disciplina;

4) Monitorar positivamente.

Para todas essas ações devem ser identificados e ensinados, concomitantemente, os componentes não-verbais (gestos, expressões faciais e corporais) e paralinguísticos (tom e forma da fala, clareza, fluência, ênfase etc) que são essenciais para uma interação eficaz com os filhos (Del Prette \& Del Prette, 2008).

A possibilidade de focalizar as habilidades pessoais e parentais em treinamentos para pais de crianças com necessidades especiais são encontrados em diversos estudos da área de habilidades sociais (Barros, 2008; Freitas, 2005; Pinheiro, 2006). Ao apontarem melhora na competência social das mães de crianças com diferentes tipos de necessidades educacionais, o resultado desses estudos fortalece essa proposta por se tratar de filhos com necessidades educacionais especiais, o que requer um repertório ainda mais elaborado dos pais para cumprirem seu papel de educadores, bem como para lidarem no seu dia-a-dia com as diferentes demandas inerentes às dificuldades da criança.

\section{CONSIDERAÇÕES FINAIS}

O grande desafio da educação inclusiva é a transformação do contexto escolar de modo que nele sejam educadas, sem distinção, todas as crianças. A concretização dessa transformação não depende apenas das políticas públicas nem somente das escolas, mas de uma parceria com todos aqueles 
envolvidos com a criança, incluindo principalmente a família. Além disso, essa transformação não pode restringir-se aos aspectos eminentemente acadêmicos, pois falar em inclusão é também valorizar as interações sociais positivas do aluno com outros participantes do contexto escolar como indicadores da efetiva inclusão escolar.

No caso de crianças com o TDAH, esses dois aspectos entrelaçam-se de uma forma bastante contundente, pois além das dificuldades em responder às demandas acadêmicas, em termos de aprendizagem, o padrão de interações sociais apresentado com os colegas e os professores está bastante prejudicado (Nixon, 2001; Hoza et al., 2005), o que torna a escolarização dessas crianças um grande desafio. Talvez por isso, como verificado por Omote (2005), os professores do ensino regular sejam mais favoráveis à inclusão de alunos com deficiência física e menos à inclusão de alunos com distúrbios emocionais e de comportamento (condutas típicas).

Frente a esse contexto, o presente ensaio foi conduzido considerando, dentre outros aspectos, o proeminente papel dos pais na inclusão social e escolar dos seus filhos e o seu acesso aos diversos contextos em que a criança participa, enquanto coadjuvantes no ensino de comportamentos que favorecem a inclusão social e escolar dessas crianças. Isso não significa reduzir o papel dos professores nessa tarefa nem imputar à família a responsabilidade por esse processo. Entende-se que a família, enquanto componente ativo, contribuinte e essencial à educação dos filhos, divide responsabilidades com a escola para enfrentar os desafios da escolarização impostos à criança com TDAH. Para essa parceria, é importante que os professores sejam cada vez mais informados sobre TDAH e que reconheçam os pais como interlocutores privilegiados e imediatos da tarefa que lhes cabe.

Com relação ao TDAH, enquanto categoria da Educação Especial, concordamos com Brand, Dunn e Greb (2002) ao defenderem que o momento atual exige a promoção de uma abordagem que seja baseada nas necessidades educacionais específicas de cada estudante com TDAH e não na categoria genérica de Educação Especial na qual o indivíduo foi classificado. Esse reconhecimento é importante visto que os alunos com TDAH, embora possam ter o mesmo diagnóstico ou características comuns, apresentam grande variabilidade na forma e no comportamento individual das crianças em vários contextos (Desidério \& Miyazaki, 2007). O respeito a essa individualidade, apesar do rótulo, pode garantir uma educação de fato especial, que considere a necessidade de cada criança ou de sua família.

\section{REFERÊNCIAS}

Adesida, O., \& Foreman, D. (1999). A support group for parents of children with hyperkinetic disorder: An empowerment model. Clinical Child Psychology and Psychiatry, 4(4), 567-578.

Banks, T., Ninowski, J. E., Mash, E. J., \& Semple, D. L. (2008). Parenting behavior and cognitions in a community sample of mothers with and without symptoms of attention deficit/hyperactivity disorder. Journal of Child \& Family Studies, 17(1), 28-43.

Barkley, R. A. (2002). Transtorno de déficit de atenção/hiperatividade (TDAH): Guia completo e autorizado para pais, professores e profissionais da saúde. Porto Alegre: Artmed.

Barros, S. K. S. N. (2008). Treinamento de habilidades sociais para pais de crianças com queixas escolares. Dissertação de Mestrado, Universidade Federal de São Carlos, São Paulo.

Brand, S., Dunn, R., \& Greb, F. (2002). Learning styles of students with attention deficit hyperactivity disorder: Who are they and how can we teach them? The Clearing House, 75(5), 268-273.

Brasil. (2001). Diretrizes nacionais para a educação especial na educação básica. Brasília: MEC/SEESP.

Brasil. (2002a). Estratégias e orientações para a educação de alunos com dificuldades de aprendizagem associadas às condutas típicas. Brasília: MEC/SEESP.

Brasil. (2002b). Projeto Escola Viva: Garantindo o acesso e permanência de todos os alunos na escola: Alunos com necessidades educacionais especiais - Reconhecendo os alunos que apresentam dificuldades acentuadas de aprendizagem relacionadas a condutas típicas (série 2). Brasília: MEC/SEESP. 
Brasil. (2006). Dados da educação especial no Brasil. Brasília: MEC/INEP. Recuperado em 10 set. 2006, em http://portal.mec.gov.br/seesp/

Chronis, A. M., Chacko, A. Fabiano, G. A., Wymbs, B. T., \& Pelham, W. E. (2004). Enhancements to the behavioral parent training paradigm for families of children with ADHD: Review and future directions. Clinical Child and Family Psychology Review, 7(1), 1-27.

Condemarín, M., Gorostegui, M. E., \& Milicic, N. (2006). Transtorno de déficit de atenção: Estratégias para o diagnóstico e a intervenção psicoeducativa. São Paulo: Planeta do Brasil.

Cunningham, C. E. (2007). A family-centered approach to planning and measuring the outcome of interventions for children with attentiondeficit/hyperactivity disorder. Ambulatory Pediatrics, 7(1S), 60-72.

Cypel, S. (2007). Déficit de atenção e hiperatividade e as funções executivas: Atualização para pais, professores e profissionais da saúde. São Paulo: Lemos.

Del Prette, A., \& Del Prette, Z. A. P. (2001). Psicologia das relações interpessoais: Vivências para o trabalho em grupo. Petrópolis: Vozes.

Del Prette, Z. A. P., \& Del Prette, A. (2005). Psicologia das habilidades sociais na infância: Teoria e prática. Petrópolis: Vozes.

Del Prette, A., \& Del Prette, Z. A. P. (2008). Pais e professores contribuindo para o processo de inclusão: Que habilidades sociais educativas devem apresentar? In M. A. Almeida; E. G. Mendes, \& M. C. P. I. Hayashi. (Org.). Temas em educação especial: Conhecimentos para fundamentar a prática (pp. 239-254). Araraquara: Junqueira \& Marin Editores.

Del Prette, A., \& Del Prette, Z. A. P. (2009). Adolescência e fatores de risco: A importância das habilidades sociais educativas. In V. G. Haase \& F. J. Penna. (Org.). Aspectos biopsicossociais da saúde na infância e adolescência (pp. 503-522). Belo Horizonte: Coopmed.

Desidério, R. C. S., \& Miyazaki, M. C. O. S. (2007). Transtorno de Déficit de Atenção/Hiperatividade (TDAH): Orientações para a família. Psicologia Escolar e Educacional, 11(1), 165-176.
DuPaul, G. J., \& Stoner, G. (2007). TDAH nas escolas: Estratégias de avaliação e intervenção. São Paulo: M. Books.

Ferreira, W. B. (2005). Será que sou a favor ou contra uma escola de qualidade para todos? Revista Inclusão, 1(1), 40-46, Brasília: MEC/SEESP.

Ferreira, W. B. (2006). Educar na diversidade: Práticas educacionais inclusivas na sala de aula regular. Ensaios pedagógicos: Programa educação inclusiva. Brasília: MEC/SEESP.

Finzi-Dottan, R., Manor, I., \& Tyano, S. (2006). ADHD, temperament, and parental style as predictors of the child's attachment patterns. Child Psychiatry and Human Development, 37, 103-114.

Freire, A. C. C., \& Ponde, M. P. (2005). Estudo piloto da prevalência do transtorno de déficit de atenção e hiperatividade entre crianças escolares na cidade de Salvador, Bahia, Brasil. Arquivos de Neuropsiquiatria, 63(2-B), 474-478.

Freitas, M. G. (2005). Desenvolvimento e avaliação de um programa de habilidades sociais com mães de crianças deficientes visuais. Tese de Doutorado. Universidade Federal de São Carlos, São Paulo.

Glat, R., \& Fernandes, E. M. (2005). Da educação segregada à educação inclusiva: Uma breve reflexão sobre os paradigmas educacionais no contexto da Educação Especial brasileira. Revista Inclusão, 1, 35-39, Brasília: MEC/SEESP.

Golfeto, J. H., \& Barbosa, G. (2003). A. Epidemiologia. In L. A Rohde \& P. Mattos (Org.). Princípios e práticas em transtorno de déficit de atenção/ hiperatividade (pp. 15-33). Porto Alegre: Artmed.

Guardiola, A., Fuchs, F. D., \& Rotta, N. T. (2000). Prevalence of attention-deficit hyperactivity disorders in students: comparison between DSMIV and neuropsychological criteria. Arquivos de Neuropsiquiatria, 58(2-B), 401-407.

Hoerger, M. L., \& Mace, F. C. (2006). A computerized test of self-control predicts classroom behavior. Journal of Applied Behavior Analysis, 39(2), 147-159.

Hoza, B., Mrug, S., Gerdes, A. C., Hinshaw, S. P., Bukowski, W. M., \& Gold, J. A. et al. (2005). What aspects of peer relationships are impaired in children with attention-deficit/hyperactivity disorder? Journal of Consulting and Clinical Psychology, 73(3), 411-423. 
Hübner, M. M., \& Marinotti, M. (2000). Crianças com dificuldades escolares. In E. F. M. Silvares. (Org.). Estudos de caso em psicologia clínica comportamental infantil (pp. 259-304). Campinas: Papirus.

Johnson, C., \& Mash, E. J. (2001). Families of children with attention-deficit/hyperactivity disorder: Review and recommendations for future research. Clinical Child and Family Psychology Review, 4(1), 183-207.

Landskron, L. M. F., \& Sperb, T. M. (2007). As narrativas dos professores sobre a experiência com o TDAH: Um estudo de caso coletivo. [Resumo]. In VI Congresso Brasileiro de Psicologia do Desenvolvimento (p. 177). Vitória: Autor. Recuperado em 18 abr. 2008, em http://www.msmidia.com/ sbpd/vi_cbpd_anais_2007.pdf

McLaughlin, D. P., \& Harrison, C. A. (2006). Parenting practices of mothers of children with ADHD: The role of maternal and child factors. Child and Adolescent Mental Health, 11(2), 82-88.

America Psychiatric Association. (2002). Manual diagnóstico e estatístico de transtornos mentais. Porto Alegre: Artmed.

Multimodal Treatment Study of Children with ADHD. (1999). A 14-month randomized clinical trial of treatment strategies for attention-deficit hyperactivity disorder (ADHD). Archives of General Psychiatry, 56(12), 1073-1086.

Neef, N. A., Marckel, J., \& Ferreri, S. J. (2005). Behavioral assessment of impulsivity: A comparison of children with and without attention deficit hyperactivity disorder. Journal of Applied Behavior Analysis, 38(1), 23-37.

Neophytou, K., \& Webber, R. (2005). Attention deficit hyperactivity disorder: The family and social context. Australian Social Work, 58(3), 313-325.

Nixon, E. (2001). The social competence of children with attention deficit hyperactivity disorder: review of the literature. Child Psychology \& Psychiatry Review, 6(4), 172-180.
Omote, S. (2005). Medida de atitudes sociais em relação à inclusão [Resumo]. In Sociedade Brasileira para o Progresso da Ciência. 57 Reunião Anual da SBPC. Fortaleza: Autor. Recuperado em 24 out. 2006, em http://www.sbpcnet.org.br/livro/57ra/programas/CONF_SIMP/textos/ sadaoomote.htm

Petersen, D. J., Bilenberg, N., Hoerder, K., \& Gillberg, C. (2006). The population prevalence of child psychiatric disorders in Danish 8 to 9-year-old children. European Child and Adolescent Psychiatry, 15(2), 71-78.

Pinheiro, M. I. S. (2006). Treinamento em habilidades sociais educativas para pais de crianças em trajetória de risco. Dissertação de Mestrado. Universidade Federal de São Carlos, São Paulo.

Poeta, L. S., \& Neto, F. R. (2004). Estudo epidemiológico dos sintomas do Transtorno do Déficit de Atenção/Hiperatividade e Transtornos de Comportamento em escolares da rede pública de Florianópolis usando a EDAH. Revista Brasileira de Psiquiatria, 26(3), 150-155.

Possa, M. de A., Spanemberg, L., \& Guardiola, A. (2005). Comorbidades do transtorno de déficit de atenção e hiperatividade em crianças escolares. Arquivos de Neuropsiquiatria, 63(2-B), 479-483.

Rielly, N. E., Craig, W. M., \& Parker, K. C. H. (2006). Peer and parenting characteristics of boys and girls with subclinical attention problems. Journal of Attention Disorders, 9(4), 598-606.

Rocha, M. M. Programa de habilidades sociais educativas com pais: Efeitos sobre o desempenho social e acadêmico de filhos com TDAH. Tese de Doutorado, Universidade Federal de São Carlos, São Paulo.

Rohde, L. A., \& Benzik, E. B. P. (1999). Transtorno de déficit de atenção e hiperatividade: $O$ que é? Como ajudar? Porto Alegre: Artes Médicas. 
Rohde, L. A., Biederman, J., Busnello, E. A., Zimmermann, H., Schmitz, M., \& Martins, S., et al. (1999). ADHD in a school sample of Brazilian adolescents: A study of prevalence, comorbid conditions, and impairments. Journal of the American Academy of Child and Adolescent Psychiatry, 38(6), 716-723.

Sena, S. S., \& Neto, O. D. (2007). Distraído e a 1000 por hora: Guia para familiares, educadores e portadores do transtorno de déficit de atenção e hiperatividade. Porto Alegre: Artmed.

Souza, I., \& Pinheiro, M. A. S. (2003). Co-morbidades. In L. A. Rohde \& P. Mattos. (Org.). Princípios e práticas em transtorno de déficit de atenção/ hiperatividade (pp. 85-105). Porto Alegre: Artmed.

Souza, I., Serra, M. A., Mattos, P., \& Franco, V. A. (2001). Comorbidade em crianças e adolescentes com transtorno do déficit de atenção: Resultados preliminares. Arquivos de Neuropsiquiatria, 59(2-B), 401-406.

Tripp, G., Schaughency, E. A., Langlands, R., \& Mouat, K. (2006). Family interactions in children with and without ADHD. Journal of Child and Family Studies, 16(3), 385-400.

Vasconcelos, M. M., Werner JR., J., Malheiros, A. F. A., Lima, D. F. N., Santos, I. S. O., \& Barbosa, J. B. (2003). Prevalência do transtorno de déficit de atenção/hiperatividade numa escola pública primária. Arquivos de Neuro-Psiquiatria, 61(1), 67-73.

Wells, K. C., Pelham, W. E., Kotkin, R. A., Hoza, B., Abikoff, H. B., Abramowitz, A, et al. (2000). Psychosocial treatment strategies in the MTA Study: Rationale, methods, and critical issues in design and implementation. Journal of Abnormal Child Psychology, 28(6), 483-505.

Recebido: 19/05/2009 Received: 05/19/2009

Aprovado: 26/08/2009 Approved: 08/26/2009 